\title{
Artificial Intelligence in Gastrointestinal Endoscopy in a Resource-constrained Setting: A Reality Check
}

\author{
Prajna Anirvan ${ }^{1}$, Dinesh Meher ${ }^{2}$, Shivaram P Singh ${ }^{3}$
}

\begin{abstract}
Artificial intelligence (AI) is being increasingly explored in different domains of gastroenterology, particularly in endoscopic image analysis, cancer screening, and prognostication models. It is widely touted to become an integral part of routine endoscopies, considering the bulk of data handled by endoscopists and the complex nature of critical analyses performed. However, the application of Al in endoscopy in resourceconstrained settings remains fraught with problems. We conducted an extensive literature review using the PubMed database on articles covering the application of Al in endoscopy and the difficulties encountered in resource-constrained settings. We have tried to summarize in the present review the potential problems that may hinder the application of Al in such settings. Hopefully, this review will enable endoscopists and health policymakers to ponder over these issues before trying to extrapolate the advancements of Al in technically advanced settings to those having constraints at multiple levels.

Keywords: Artificial intelligence, Automated detection, Computer-aided detection, Deep learning, Developing countries, Lesion detection, Health resources, Health services accessibility.

Euroasian Journal of Hepato-Gastroenterology (2020): 10.5005/jp-journals-10018-1322
\end{abstract}

"Good afternoon... gentlemen. I am a HAL 9000... computer. I became operational at the H.A.L. plant [voice becomes lower \& slower] in Urbana, Illinois... on the 12th of January 1992. [voice becomes even more lower \& slower] My instructor was Mr. Langley... and he taught me to sing a song. If you'd like to hear it, I can sing it for you."

\section{- HAL 9000 (2001: A Space Odyssey)}

\section{INTRODUCTION}

Artificial intelligence (Al), a milestone in humankind's scientific achievements, is an application of computer science based on mathematical logic that utilizes computers to imitate learning, memorizing, and analytical reasoning-attributes traditionally associated with human intelligence and cognition. ${ }^{1}$ The idea of $\mathrm{Al}$ is not new. We find references in fictional characters-Mary Shelly's Frankenstein and Arthur Clarke's HAL 9000 being two fine examples. The mathematician Alan Turing is generally credited with conceptualizing Al. Artificial intelligence as a discipline of research came into being in 1956 when John McCarthy coined the term "Artificial Intelligence." ${ }^{2}$ In healthcare, Al found its first application in the domain of diagnostics as a medical diagnostic decision support (MDDS) system. ${ }^{3}$ Research in the sixth and seventh decades of the last century resulted in the advent of Dendral, the first program to solve problems. ${ }^{4}$ Although originally it was meant to be applied in organic chemistry, it laid the groundwork for another system, MYCIN, which was a very significant advancement in the use of Al in healthcare. ${ }^{5}$ In 1969, De Dombal et al. designed a very effective MDDS system for clinical diagnosis. ${ }^{6}$ Soon enough, MDDS systems were being applied in multiple disciplines: forensic medicine, internal medicine, pathology, radiodiagnosis, and psychiatry. In the days to come, Al technology is envisaged to play a decisive role in diagnosis and treatment, maintenance of health records, and overall functioning of healthcare systems including diagnosis, treatment, patient care, and other upcoming areas in medicine. ${ }^{7}$ Gastroenterology is one
${ }^{1-3}$ Department of Gastroenterology, SCB Medical College, Cuttack, Odisha, India

Corresponding Author: Shivaram P Singh, Department of Gastroenterology, SCB Medical College, Cuttack, Odisha, India, Phone: +91 6712505466, e-mail: scb_gastro_dept@hotmail.com

How to cite this article: Anirvan P, Meher D, Singh SP. Artificial Intelligence in Gastrointestinal Endoscopy in a Resource-constrained Setting: A Reality Check. Euroasian J Hepato-Gastroenterol 2020;10(2): 92-97.

Source of support: Kalinga Gastroenterology Foundation, Cuttack, India. Conflict of interest: None

such branch where clinical decision making and treatment are driven by complex endoscopic procedures, maneuvers, and visual identification-cum-interpretation that require a lot of technical skill and expertise. Clinicians, in gastroenterology, come across huge amounts of data and a myriad of endoscopic images. This is where Al can be used to aid gastroenterologists in endoscopic diagnosis, image analysis, screening of malignancies and other lesions, and also in developing prognostic models.

\section{Al-Machine Learning and Deep Learning}

Machine learning (ML) and deep learning (DL) can be considered subsets of Al (Fig. 1). Machine learning, an elemental concept in $\mathrm{Al}$ ever since the inception of the discipline, can be described as the study of computer algorithms, which over a period of time through training and practice, improve automatically. ${ }^{8}$ Based on sample data, otherwise called "training data," ML algorithms devise mathematical models which makes it possible to predict and take decisions without explicit programming. Machine learning is again subdivided into supervised learning and unsupervised learning. In the former, the computer is presented with sample inputs along with the outputs that are desired and are taught the general rule of mapping these 


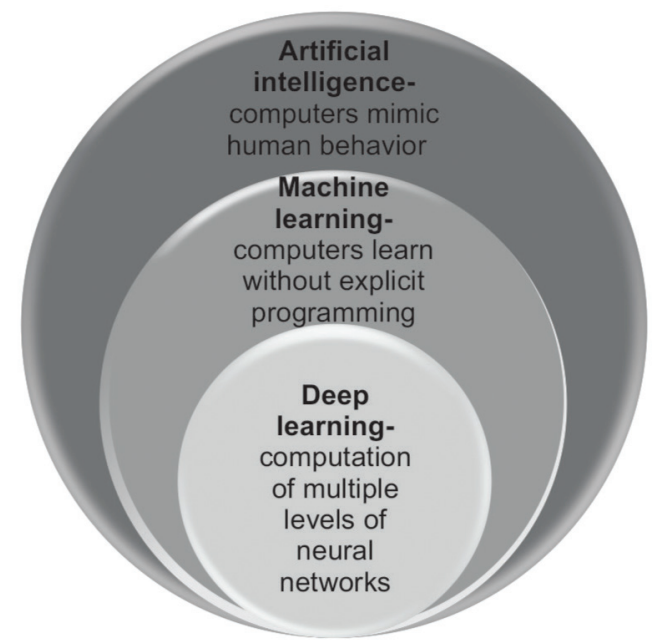

Fig. 1: $M L$ and $D L-$ subsets of $A I$

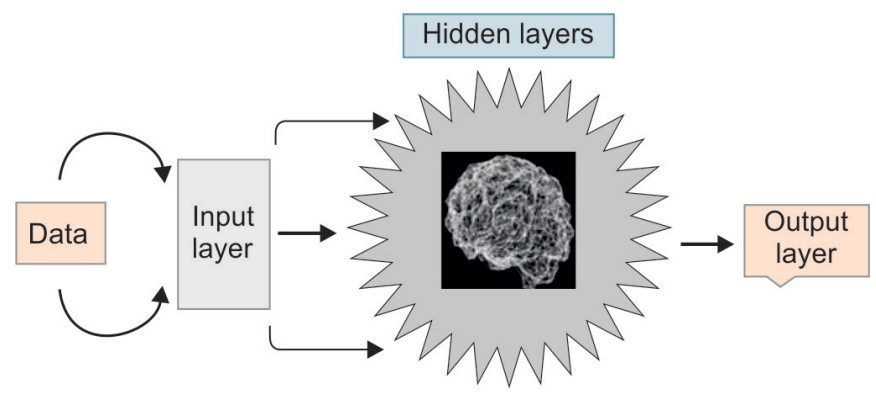

Fig. 2: Deep learning - a diagrammatic representation

inputs to outputs. In the latter, the learning algorithm is left to itself to recognize a structure in its input sample. Predictive models are fashioned by the supervised ML algorithm which allows new inputs to be mapped to outputs. ${ }^{9}$ Artificial neural networks (ANN) are supervised models quite akin to the organizational structure of the human central nervous system. Neurons are computing units and are interconnected to form a network. From the input layer, a signal traverses through numerous hidden layers en route to the output layer. ANN training encompasses separating the data into a "training set" to define the network architecture and a "test set" to evaluate the ability of ANN to predict the desired output. The quest for improved performance has resulted in the development of increasingly complex neural networks, leading to the concept of DL. ${ }^{10}$ Deep learning uses multiple layers to progressively extract features of a higher-level from the raw input. A deep neural network (DNN) consists of multiple consecutive filters that enable the automatic detection of important characteristics of input data. However, for improved performance, an enormous amount of labeled training data is required which has led to the combining of DL with reinforcement learning principles. ${ }^{11}$ An example of the use of the application of DL technology is the training of a deep convolutional neural network (CNN) on 129,450 dermatological images consisting of 2,032 different skin disorders the performance of which was comparable to 21 board-certified dermatologists in differentiating keratinocyte carcinomas from benign seborrheic keratosis and malignant melanomas from benign nevi. ${ }^{12}$ Figure 2 depicts a diagrammatic representation of the concept of $\mathrm{DL}$.

\section{Gastrointestinal (GI) Endoscopy and Al Application: An Ever-widening Spectrum}

The applications of $\mathrm{Al}$ in the various domains of $\mathrm{Gl}$ endoscopy are manifold. A computer algorithm trained to perform specific functions like recognizing or characterizing defined lesions lies at the heart of Al. We summarize here the major areas where the application of Al has been found to be useful both for diagnostic and prognostic purposes.

\section{Image Analysis}

Globally, GI malignancies account for $26 \%$ of cancer incidence and $35 \%$ of cancer-related mortality. ${ }^{13}$ In 2018 , the number of new cases of $\mathrm{Gl}$ cancer worldwide was 4.8 million while cancer-related deaths amounted to 3.4 million. ${ }^{13}$ In a bid to increase the rate of detection of gastrointestinal neoplasms and strengthen screening programs, accurate endoscopic examination and proper differentiation of benign from malignant lesions are essential. As previously discussed, training of a computer algorithm is performed using ML by exposing it to training elements, for example, a huge number of predefined video frames depicting various polyps. The algorithm aids conventional endoscopy by extracting and analyzing specific features: the topological pattern of the polyp surface, variation in color, microvasculature, or appearance under narrow-band imaging (NBI), high-magnification, and endocystoscopy, which translates into enhanced and improved quality of lesion detection and prediction of diagnosis. ${ }^{14}$ Subsequently, a different test database is employed to validate this algorithm. A fine example of such an application is the demonstration by Horie et al. that diagnosis of esophageal cancer could be made using CNN trained with 8,428 images obtained from conventional endoscopy that included white-light images (WLIs) and narrow-band images (NBIs). ${ }^{15}$ The sensitivity of esophageal cancer detection was $95 \%$, and even small malignancies of $<10 \mathrm{~mm}$ could be detected. Besides, superficial esophageal cancer could also be differentiated from advanced malignancy with 98\% accuracy. ${ }^{15}$ In addition to the detection of lesions, the characterization of the lesion has also been done with the help of Al. Application of the CNN system to assess the invasive depth of carcinoma stomach using conventional endoscopic images by Zhu et al. demonstrated high accuracy (89.2\%) and specificity (95.6\%) which was considerably better compared to experienced endoscopists. ${ }^{16}$ The results of Al application in the detection and characterization of colonic polyps have also been very encouraging. A CNN model by Urban et al. resulted in real-time polyp detection with an AUROC of 0.991 and 96.4\% accuracy. ${ }^{17}$ Capsule endoscopy, despite providing a means to evaluate and explore small bowel lesions has disadvantages, such as the low quality of images generated and the fact that interpretation of images is highly subjective. Here too, CNN has shown immense promise. In a study by Leenhardt et al., a model to detect $\mathrm{Gl}$ angiectasia designed using CNN demonstrated improved performance with 100\% sensitivity, $96 \%$ specificity, 96\% PPV, and $100 \%$ NPV. ${ }^{18}$ Application of DL in WCE has also been shown to facilitate detection of the bleeding source from the small bowel. Methods in Computer-Aided Detection (CADe) that have evolved have a likelihood of being affected by movements of the camera, optical disturbances pertaining to light reflection and focus of the lens, variability in the morphology of lesions, and distractors like feces, bubbles, etc. ${ }^{19}$ To overcome these barriers, CADe systems are being developed where context information is utilized and nonpolypoid lesions or structures are removed from the analysis and shape information is utilized to aid in polyp localization. As a 


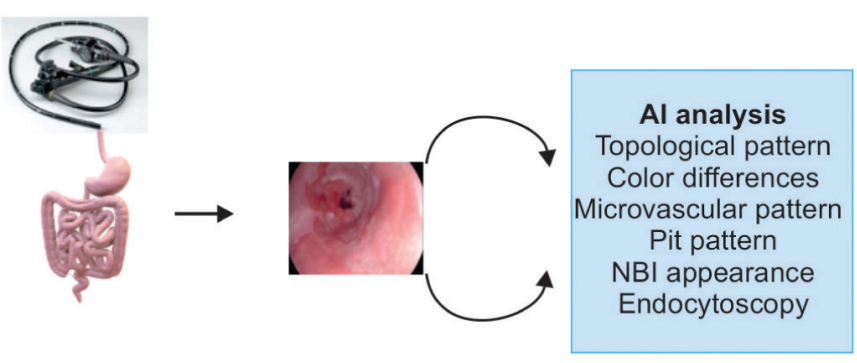

Fig. 3: Image analysis using Al

further modification and advancement to this technique, real-time polyp detection modalities on larger colonoscopy image databases are also coming up. Even in Helicobacter pylori infection diagnosis, Al has been shown to be helpful. A CNN model was developed by Itoh et al. to aid in recognition of $H$. pylori infection which demonstrated encouraging results with a sensitivity of $86.7 \%$ and specificity of $86.7 \% .^{20}$ Figure 3 shows the process of image analysis using $\mathrm{Al}$ in endoscopy.

\section{Optical Biopsy}

The next step after lesion detection is to assess its nature, which traditionally requires a tissue biopsy. However, computational analysis, otherwise called computer-aided diagnosis (CADx), may assist in the prediction of histology even without a tissue biopsy. Optical biopsy can diagnose adenoma in situ and enable early resection obviating the need for unnecessary histopathologic examination. ${ }^{21}$ Narrow-band imaging and chromoendoscopy are also subject to considerable interobserver and intraobserver variability. Using CADx modalities may help decrease interobserver variance, increase standardization, and make possible more extensive adoption by nonexperts in the field. ${ }^{22}$ CAD-aided endocytoscopy has been developed that makes use of nuclear segmentation and feature extraction to assist in pathologic classification (i.e., differentiating among non-neoplastic, adenomatous, and malignant lesions). ${ }^{23}$

\section{Diagnosis Making and Prediction of Prognosis}

Apart from the utility of $\mathrm{Al}$ in image analysis, various $\mathrm{ML}$ models have demonstrated encouraging results in making diagnosis and forecasting prognosis. In stark contrast to the human brain which has limited capabilities in its ability to handle large volumes of data, ANN has the ability to analyze such complex datasets and handle complicated interactions among clinical, environmental, and demographic variables. An ANN model has been developed which can diagnose gastroesophageal reflux disease using just 45 clinical variables extracted from 159 cases with $100 \%$ accuracy. ${ }^{24}$ In another study, recognition of atrophic gastritis was done merely by using clinical and biochemical variables from 350 outpatients with the help of ANNs and linear discriminant analysis with great accuracy. ${ }^{25}$

In prognostication, too, Sato et al. designed an ANN model to predict 1-year and 5-year survival rates based on 418 patients with carcinoma esophagus. Compared to conventional linear discriminant analysis, it yielded greater accuracy. ${ }^{26}$ In another study concerning prognostication utilizing ANN, Das et al. made a comparison between ANN performance and a scoring system termed "BLEED" which had been validated earlier. This study revealed significantly greater predictive accuracy for several prognostic indicators in the ANN model, especially mortality
( $87 \%$ vs $21 \%$ ), recurrent bleeding ( 89 vs $41 \%$ ), and the requirement for therapeutic intervention ( 96 vs $46 \%$ ). ${ }^{27}$ Further evidence of the utility of ANN is provided in the study by Rotondano et al. in which the Rockall score was compared to an ANN model using 2380 patients to predict mortality in nonvariceal upper gastrointestinal bleeding. ${ }^{28}$ This ANN model demonstrated greater sensitivity, specificity, and accuracy compared to the complete Rockall score. ${ }^{28}$

\section{Al in Resource-constrained Settings: Potential Bottlenecks \\ Al Cost-effectiveness and Economic Impact: Blue are the Hills that are Far Away?}

The application of Al in gastroenterology and Gl endoscopy, in particular, promises a lot in the days to come. Having said that, it is important to take note of the fact that not many researchers have looked into the economic aspect of Al. In the era of value-based healthcare we are living in, and also because of the high share of the healthcare industry in the overall economy, economic impact assessment is of increasing importance. ${ }^{29}$ This assumes even greater significance when applying such technology in resourceconstrained settings. This, therefore, warrants proper analyses vis-à-vis cost-effectiveness which needs to be optimized in a resource-constrained setting. The $\mathrm{Al}$ application has been widely touted to drastically slash healthcare expenditures and costs. However, Al application in a field like gastroenterology, especially gastrointestinal endoscopy in a resource-constrained setting and its economic impact warrants close scrutiny.

For rural clinics without physicians in India, a computer-assisted diagnostic system, early detection and prevention system (EDPS) was developed. Appropriate guidance and recommendations for nurses and other paramedical staff were provided through this system. The overall rate of consistency between physicians and EDPS was found to be $94 \%$ based on 933 patients in a study at the Kempegowda Institute of Medical Science in Bangalore, India. ${ }^{3}$ Also, patient responses were found to be positive in another study, as patients believed that the computer-based system was superior and there was better in-depth interaction with them compared to healthcare personnel. ${ }^{30}$ Studies and reports from sub-Saharan Africa and China have also reported similar findings. ${ }^{31,32}$ Thus, medical Al technology has been envisaged to improve upon the efficiency of doctors and healthcare service quality, bring about a reduction in medical costs, and also to train nurses and paramedical health workers in areas that lack doctors, thus greatly reducing healthcare costs. ${ }^{3}$

While the above findings may look very encouraging, a closer look at the healthcare system in a country like India, a "developing" country with multiple constraints in its healthcare system would put things in perspective. With its resource-constrained settings, despite healthcare being a growing industry, valued at nearly $\$ 40$ billion, ${ }^{33}$ there are challenges in ensuring equitable healthcare to all. A major challenge is that healthcare spending in India is largely out of pocket, with almost $70 \%$ of hospitals and $40 \%$ of hospital beds being private. ${ }^{33}$ Healthcare in India has come a long way since independence and has had remarkable success in various important health indices. However, there remains much to be done with palpable weaknesses in organization, funding, and provision of health services. ${ }^{34}$ Health insurance is, to a large extent, private, and the poor have limited access to private care. Healthcare expenditure remains at only $4.1 \%$ of gross domestic product (GDP), and there is a huge disparity between rural and urban regions in India as regards provisioning of resources. ${ }^{33}$ 
Employment State Insurance Scheme (ESIS) and Central Government Health Scheme (CGHS) are two major state health insurance schemes in India catering to factory workers and employees of the central government, respectively. ${ }^{35}$ The Government of India has initiated other national health insurance schemes like the Rashtriya Swasthya Bima Yojana, Universal Health Insurance Scheme, Aam Aadmi Bima Yojana, Janashree Bima Yojana, and very recently, the Ayushman Bharat scheme. However, despite attempts to expand social healthcare insurance, a large informal sector, inadequacies in understanding solidarity-based insurance, lack of data on costing, free and unregulated private market, and low standards of public healthcare delivery have complicated things. ${ }^{36}$ In other developing countries too, the narrative is more or less the same, poor spending on public health, inadequate health insurance, restricted benefit packages, the paucity of health professionals and facilities, deficiencies in training of health workers, and transportation difficulties had resulted in poor quality of healthcare in the rural areas. ${ }^{37}$

Looking at the advancement of Al technology over the years, and the application of such technology in various aspects of healthcare, it can be extrapolated that for Al applications in the various domains of gastroenterology, certain prerequisites are essential. Firstly, an Al-based clinical decision support system that is both practical and economical must be in place. This system should focus primarily on common gastrointestinal disorders and diseases that are amenable to screening programs. Secondly, the affordability of such a model must be kept in mind, considering the healthcare structure of developing countries. Thirdly, the infrastructure needed for procuring and maintaining the facility has to be developed. For example, in addition to endoscopes, there has to be an adequate number of computers (desktops and laptops), appropriate software, uninterrupted power supply, etc. There has to be proper connectivity for the transmission of information. And most importantly, one of the basic prerequisites is the training of doctors, healthcare workers, and other personnel in the different modalities of $\mathrm{Al}$ application. ${ }^{3}$

Although there are no studies that have directly looked into the cost-effectiveness of $\mathrm{Al}$ in $\mathrm{Gl}$ endoscopy in a resource-constrained setting, a study by Dalaba et al. which investigated the financial aspects regarding the implementation of a computer-assisted clinical decision support system for antenatal and peripartum care in Northern Ghana can be examined as a test example. ${ }^{38}$ For each healthcare worker trained, the total financial cost amounted to around $\$ 1060$ (roughly $68,772.8$ INR according to existing exchange rates), of which the cost of equipment accounted for the highest proportion of the financial cost. Cost pertaining to personnel accounted for $28.6 \%, 12.1 \%$ for meeting and training, cost relating to transportation accounted for $8.5 \%$, and other costs amounted to $16.8 \% .{ }^{38}$ The same can be said for the various domains of gastroenterology and Gl endoscopy in which $\mathrm{Al}$ is supposed to play a major role. While optical biopsy and image analysis sound fine, the cost that would be incurred for these techniques remains an area of concern. While in developed countries with a well-equipped healthcare model in place this may not be a problem, in poor, rural, and resource-constrained settings, it may aggravate the burdened healthcare system in place. Hence, validation of cost-effectiveness is necessary before developing and designing any such Al program. Reasonable regulations also need to be commissioned by appropriate regulatory authorities while provisions must be made for reimbursement before integrating Al technology in the field of $\mathrm{Gl}$ endoscopy. In gastroenterology setups in government-run medical colleges and hospitals which cater to the bulk of the population, without addressing the problems of health insurance, equitable healthcare, and maintenance of equipment, Al application may well turn out to be the white elephant of Gl endoscopy.

\section{Technical Concerns}

In developing countries like India, the Gl endoscopy centers both in government hospitals and private centers are manned, in addition to endoscopists, by nurses and technicians. The Al programs that are designed are meant for adequately trained doctors. Without making user-friendly programs and educating the paramedics and healthcare workers, the application of Al will not serve its purpose. To add to this, regional medical Al support centers have to be established that will oversee the entire Al network and will carry out periodic inspection, assessment, and upgradation of the existing system.

The medical colleges and government-run hospitals that have dedicated departments of gastroenterology generally cater to a large population from the rural areas and a huge number of endoscopic procedures are done daily. Often, it is seen that there is a lack of adequate staffing and infrastructure in endoscopy suites and clinicians have to work day in and out under extremes of professional constraints. In such circumstances, the "imposition" of Al-based technology in the field of Gl endoscopy might create greater confusion and may turn out to be an additional burden, ultimately failing to live up to its primary purpose.

\section{Professional Issues: Gastroenterologists Reduced to Mere Technicians!}

In addition to the technical training and know-how that has to be imparted to physicians, regular system upgrades are also required to keep pace with the latest advances. It has been shown that there has been disagreement among healthcare providers with the recommendations made by medical Al devices. ${ }^{31}$ Therefore, regular training and assessment of physicians and healthcare providers to keep them abreast of the latest advances in technology are essential to prevent misuse and mismanagement of Al systems.

Some reports have shown that Al-related technologies in medicine could impair the efficacy of patient consultation which may lead to anomalous situations, such as missing out important clinical signs while focusing more on technological appliances. ${ }^{39}$ While a machine-based intelligence system can definitely outperform a human brain in terms of compiling data and interpreting it, certain features exclusively "human" are an integral part of the doctor and patient relationship. Therefore, in developing countries that are technically backward and are still largely dependent on the traditional face-to-face doctor-patient interaction, this might have a negative bearing on the overall doctor and patient relationship. In a bid to promote $\mathrm{Al}$, the gastroenterologist runs the risk of being reduced to a mere automaton, relying solely on the report generated by $\mathrm{Al}$, which in turn, might prove costly to patients and the healthcare system in general.

\section{Safety and Accountability: Everybody's Responsibility is Nobody's Responsibility?}

It has been argued by proponents of Al technology that it has the ability to cause reductions in unwarranted variations and improve the standards of quality of all endoscopists to the very best, an example being the improvement of detection rates of adenoma or carcinoma while performing colonoscopy. However, it can also be equally argued that $\mathrm{Al}$ is not fool-proof and mistakes could occur. 
The inability of algorithms to arrive at decisions based on data fed into the system ignoring contextual information and bypassing clinical judgment might prove disastrous. ${ }^{40}$ Also, automation bias could compound algorithmic errors, where Al decisions might be favored by clinicians even though these are incorrect. ${ }^{41}$ The problem of accountability regarding medical decisions involving Al has been a subject of intense debate. If, during Al-aided endoscopic image analysis, a lesion is wrongly deemed malignant and treatment regimens are instituted accordingly, who would take the responsibility? Should the endoscopist or the oncologist or the hospital or institution bear the responsibility? Or should it be the one who devised the algorithm, the vendor responsible for the deployment, or the organization providing the training data? ${ }^{42}$ In such cases concerning legal and ethical problems, who would be held accountable? In resource-constrained settings of developing countries, with a large chunk of the population unaware of the ethical issues of advanced technology in medicine, indiscriminate application of Al without addressing these issues might cause immense harm to unsuspecting patients and reduce them to mere guinea pigs in an experimental cauldron of Gl endoscopy.

\section{Data Sourcing and Validation in Real-world Setting}

Despite the reported high level of performance in the various studies concerning $\mathrm{Al}$ application in endoscopy, there remain several questions as the various proposed models have been tested only in research settings. In a "real-world" setting, for example, studying the prevalence of colorectal polyps in an Indian population with its inherent enormous amount of diversity, these very models may display different behavior when applied and have every chance of poor generalization to different populations and regions. Therefore, rigorous validation is essential to design an algorithm that can be used in a clinical setting. This includes both internal and external validation along with validation in a prospective clinical trial which, again, is time-consuming and expensive. ${ }^{43}$

Figure 4 shows the potential problems in implementing $\mathrm{Al}$ in endoscopy in a resource-constrained setting.

\section{CONCLUSION}

The Al-based application holds immense promise in various fields of Gl endoscopy. It has the potential to revolutionize the way endoscopies are being done and interpreted. Advances in deep learning techniques will definitely bring about a sea change in the

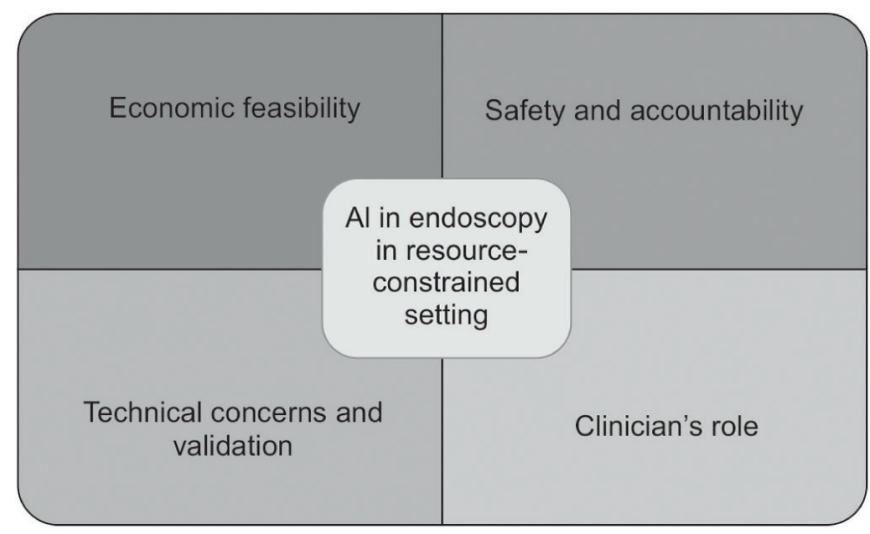

Fig. 4: Potential problems in implementing $\mathrm{Al}$ in endoscopy in a resource-constrained setting realm of image analysis and will push the borders of the existing methods of diagnosis and prognosis. While in developed countries, perhaps the revolution has already begun, in resource-constrained settings, implementation of Al in "real-world" clinical practice overcoming economic, social, and ethical barriers will prove to be a Herculean task. Simply extrapolating the results obtained from experiences in technologically advanced settings to resourceconstrained environments will not yield favorable results. Without developing healthcare infrastructure, initiating better healthcare insurance schemes, and engaging in rigorous clinical studies, Al-based technology in Gl endoscopy may probably be reduced to something of only ornamental significance. To properly utilize the immense power of $\mathrm{Al}$, there has to be investment aimed at developing the infrastructure along with the implementation of government policies that promote innovation while taking into account cost analysis and patient safety. ${ }^{42}$

\section{EPILOGUe}

In his article "The Implausibility of Intelligence Explosion" Google engineer François Chollet remarks that artificial intelligence, and for that matter all intelligence, is "fundamentally situational". ${ }^{44} \mathrm{~A}$ computer algorithm's intelligence in the endoscopic interpretation of a lesion (benign vs malignant) concerns solving the problem associated with applying that algorithm to analyze the specific data fed into it, merely adaptive to the situation it is in. It has no knowledge of the patient's vitals, functional status, and emotional state. It simply is not its concern. In a typical "real-world" resourceconstrained setting, where despite a lack of infrastructure and below-average healthcare standards, a gastroenterologist by virtue of his/her clinical acumen, humane approach, and endoscopic skills succeeds in providing reasonably affordable patient care, it would be worthwhile to remind ourselves that imposing $\mathrm{Al}$, without taking into account the existing problems, could turn into a Frankenstein because of sheer human impudence and irrationality.

\section{ACKNOWLedgment}

This work was partially supported by a grant from the Kalinga Gastroenterology Foundation, Cuttack, India.

\section{References}

1. Poole DL, Mackworth AK, Goebel R. Computational intelligence: a logical approach. New York: Oxford University Press; 1998. 558 p.

2. Rajaraman V. JohnMcCarthy - father of artificial intelligence. Resonance 2014 Mar;19(3):198-207.

3. Guo J, Li B. The application of medical artificial intelligence technology in rural areas of developing countries. Health Equity 2018 Aug 1;2(1):174-181. DOI: 10.1089/heq.2018.0037.

4. Lindsay RK, Buchanan BG, Feigenbaum EA, et al. DENDRAL: A case study of the first expert system for scientific hypothesis formation. Artif Intell 1993 Jun;61(2):209-261. DOI: 10.1016/00043702(93)90068-M.

5. Shortliffe EH. Chapter 1 - Introduction. In: Shortliffe EH, ed. computerbased medical consultations: mycin [internet]. Elsevier; 1976 [cited 2020 Jun 8]. p. 1-61. Available from: http://www.sciencedirect.com/ science/article/pii/B978044400179550007X.

6. De Dombal FT, Hartley JR, Sleeman DH. A computer-assisted system for learning clinical diagnosis. Lancet 1969 Jan 18;293(7586):145-148. DOI: 10.1016/S0140-6736(69)91149-0.

7. Jiang $\mathrm{F}$, Jiang $\mathrm{Y}$, Zhi H, et al. Artificial intelligence in healthcare: past, present and future. Stroke Vasc Neurol 2017 Jun 21;2(4):230-243. DOI: 10.1136/svn-2017-000101. 
8. Turing AM. I.-computing machinery and intelligence. Mind 1950 Oct 1;LIX(236):433-460. DOI: 10.1093/mind/LIX.236.433.

9. Shalev-Shwartz S, Ben-David S. Understanding machine learning: from theory to algorithms. USA: Cambridge University Press; 2014.

10. LeCun Y, Bengio Y, Hinton G. Deep learning. Nature 2015 May;521(7553):436-444. DOI: 10.1038/nature14539.

11. Mahmud M, Kaiser MS, Hussain A, et al. Applications of deep learning and reinforcement learning to biological data. IEEE Trans Neural Netw Learn Syst 2018;29(6):2063-2079. DOI: 10.1109/TNNLS.2018.2790388.

12. Esteva A, Kuprel B, Novoa RA, et al. Dermatologist-level classification of skin cancer with deep neural networks. Nature 2017 Feb 2;542(7639):115-118. DOI: 10.1038/nature21056.

13. Arnold M, Abnet CC, Neale RE, et al. Global burden of 5 major types of gastrointestinal cancer. Gastroenterology 2020 Jul;159(1):335-349. DOI: 10.1053/j.gastro.2020.02.068.

14. El Hajjar A, Rey J-F. Artificial intelligence in gastrointestinal endoscopy: general overview. Chin Med J (Engl) 2020 Feb 5;133(3):326-334. DOI: 10.1097/CM9.0000000000000623.

15. Horie Y, Yoshio T, Aoyama K, et al. Diagnostic outcomes of esophageal cancer by artificial intelligence using convolutional neural networks. Gastrointest Endosc 2019 Jan;89(1):25-32.DOI: 10.1016/j.gie.2018.07.037.

16. Zhu Y, Wang Q-C, Xu M-D, et al. Application of convolutional neural network in the diagnosis of the invasion depth of gastric cancer based on conventional endoscopy. Gastrointest Endosc 2019 Apr;89(4):806815.e1. DOI: 10.1016/j.gie.2018.

17. Urban G, Tripathi P, Alkayali T, et al. Deep learning localizes and identifies polyps in real time with $96 \%$ accuracy in screening colonoscopy. Gastroenterology 2018 Oct 1;155(4):1069-1078.e8. DOI: 10.1053/j.gastro.2018.06.037.

18. Leenhardt R, Vasseur P, Li C, et al. A neural network algorithm for detection of $\mathrm{Gl}$ angiectasia during small-bowel capsule endoscopy. Gastrointest Endosc 2019 Jan;89(1):189-194. DOI: 10.1016/j. gie.2018.06.036.

19. Wang Y, Tavanapong W, Wong J, et al. Polyp-Alert: near real-time feedback during colonoscopy. Comput Methods Programs Biomed. 2015 Jul;120(3):164-179. DOI: 10.1016/j.cmpb.2015.04.002.

20. Itoh $\mathrm{T}$, Kawahira H, Nakashima $\mathrm{H}$, et al. Deep learning analyzes Helicobacter pylori infection by upper gastrointestinal endoscopy images. Endosc Int Open 2018 Feb;6(2):E139-E144. DOI: 10.1055/s0043-120830.

21. Wilson Al, Saunders BP. New paradigms in polypectomy: resect and discard, diagnose and disregard. Gastrointest Endosc Clin N Am 2015 Apr;25(2):287-302. DOI: 10.1016/j.giec.2014.12.001.

22. Byrne MF, Shahidi N, Rex DK. Will computer-aided detection and diagnosis revolutionize colonoscopy? Gastroenterology 2017 Dec;153(6):1460-1464.e1. DOI: 10.1053/j.gastro.2017.10.026

23. Mori Y, Kudo S-E, Wakamura K, et al. Novel computer-aided diagnostic system for colorectal lesions by using endocytoscopy (with videos). Gastrointest Endosc 2015 Mar;81(3):621-629. DOI: 10.1016/ j.gie.2014.09.008.

24. Pace F, Buscema M, Dominici $P$, et al. Artificial neural networks are able to recognize gastro-oesophageal reflux disease patients solely on the basis of clinical data. Eur J Gastroenterol Hepatol 2005 Jun;17(6):605-610. DOI: 10.1097/00042737-200506000-00003.

25. Lahner E, Grossi E, Intraligi M, et al. Possible contribution of advanced statistical methods (artificial neural networks and linear discriminant analysis) in recognition of patients with suspected atrophic body gastritis. World J Gastroenterol 2005 Oct 7;11(37):5867-5873. DOI: 10.3748/wjg.v11.i37.5867.

26. Sato F, Shimada Y, Selaru FM, et al. Prediction of survival in patients with esophageal carcinoma using artificial neural networks. Cancer 2005 Apr 15;103(8):1596-1605. DOI: 10.1002/cncr.20938.

27. Das A, Ben-Menachem T, Cooper GS, et al. Prediction of outcome in acute lower-gastrointestinal haemorrhage based on an artificial neural network: internal and external validation of a predictive model. Lancet 2003 Oct 18;362(9392):1261-1266. DOI: 10.1016/S0140-6736(03)14568-0.
28. Rotondano G, Cipolletta L, Grossi E, et al. Artificial neural networks accurately predict mortality in patients with nonvariceal upper Gl bleeding. Gastrointest Endosc 2011 Feb;73(2):218-226, 226.e1-2. DOI: 10.1016/j.gie.2010.10.006

29. Wolff J, Pauling J, Keck A, et al. The economic impact of artificial intelligence in health care: systematic review. J Med Internet Res 2020 Feb;22(2):e16866. DOI: 10.2196/16866.

30. Friedman E. Computer-assisted medical diagnosis for rural SubSaharan Africa. IEEE Technol Soc Mag. 2009;28(3):18-27. DOI: 10.1109/ MTS.2009.934143.

31. Adepoju I-OO, Albersen BJA, De Brouwere V, et al. mHealth for clinical decision-making in Sub-Saharan Africa: a scoping review. JMIR MHealth UHealth. 2017 Mar 23;5(3):e38. DOI: 10.2196/mhealth.7185.

32. How technology is helping to improve health care in rural China [Internet]. South China Morning Post. 2018 [cited 2020 Oct 24]. Available from: https://www.scmp.com/tech/article/2135880/lookhow-china-using-technology-improve-rural-access-quality-healthcare.

33. Nair SR. Relevance of health economics to the Indian healthcare system: a perspective. Perspect Clin Res. 2015 Oct 1;6(4):225-226. DOI: 10.4103/2229-3485.167095.

34. Berman P, Ahuja R, Tandon A, et al. Government health financing in India : challenges in achieving ambitious goals. 2010 [cited 2020 Jun 8]; Available from: https://agris.fao.org/agris-search/search. do?recordID=US2014605182.

35. Ellis RP, Alam M, Gupta I. Health insurance in India: prognosis and prospectus, Economic and Political Weekly, 200035(4):207-217.J Health Manag 2001 Apr 1;3(1):141-141. DOI: 10.1177/097206340100300108.

36. Prinja S, Kaur M, Kumar R. Universal Health Insurance in India: ensuring equity, efficiency, and quality. Indian J Community Med $2012 \mathrm{Jul}$ 1;37(3):142-149. DOI: 10.4103/0970-0218.99907.

37. Strasser R, Kam SM, Regalado SM. Rural Health Care Access and Policy in Developing Countries. Annu Rev Public Health 2016;37:395-412. DOI: 10.1146/annurev-publhealth-032315-021507.

38. Dalaba MA, Akweongo P, Williams J, et al. Costs associated with implementation of computer-assisted clinical decision support system for antenatal and delivery care: case study of Kassena-Nankana district of northern Ghana. PLoS One. 2014 Sep 2;9(9):e106416. DOI: 10.1371/journal.pone.0106416.

39. Vedanthan R, Blank E, Tuikong N, et al. Usability and feasibility of a tablet-based Decision-Support and Integrated Record-keeping (DESIRE) tool in the nurse management of hypertension in rural western Kenya. Int J Med Inform 2015 Mar;84(3):207-219. DOI: 10.1016/j.ijmedinf.2014.12.005.

40. Caruana R, Lou Y, Gehrke J, et al. Intelligible models for healthcare: predicting pneumonia risk and hospital 30-day readmission. In: Proceedings of the 21th ACM SIGKDD International Conference on Knowledge Discovery and Data Mining [Internet]. Sydney, NSW, Australia: Association for Computing Machinery; 2015 [cited 2020 Jun 8]. p. 1721-1730. (KDD '15). Available from: https://doi. org/10.1145/2783258.2788613.

41. Lyell $D$, Coiera E. Automation bias and verification complexity: a systematic review. J Am Med Inform Assoc 2017 Mar 1;24(2):423-431. DOI: 10.1093/jamia/ocw105.

42. Ahmad OF, Stoyanov D, Lovat LB. Barriers and pitfalls for artificial intelligence in gastroenterology: ethical and regulatory issues. Tech Innov Gastrointest Endosc 2020 Apr 1;22(2):80-84. DOI: 10.1016/j. tgie.2019.150636.

43. Adadi A, Adadi S, Berrada M. Gastroenterology meets machine learning: status quo and quo vadis [Internet]. Vol. 2019, Advances in Bioinformatics. Hindawi; 2019 [cited 2020 Jun 8]. p. e1870975. Available from: https://www.hindawi.com/journals/abi/2019/1870975/.

44. Chollet F. The implausibility of intelligence explosion [Internet]. Medium. 2018 [cited 2020 Jun 8]. Available from: https://medium. com/@francois.chollet/the-impossibility-of-intelligence-explosion5be4a9eda6ec. 